\title{
Accessing Manufacturing Yield for Gamma Wafer Sawing Processes in COG Packaging
}

\author{
Wen-Lea Pearn, Yu-Ting Tai, Kai-Bin Huang, and Pin-Lun Ku
}

\begin{abstract}
The technology of thin film transistor liquid crystal display (LCD) has become more popular due to great demand for worldwide consumer electronic products. Driver integrated circuit (IC) is a critical device that is embedded sophisticated circuits to drive panels. Since narrow border design on display products is current trend, dimensions of driver ICs are shrunken. In the high-density LCD driver ICs, the operation of wafer sawing is essential and needs accurate yield assessment. However, inevitable process variance changes could arise from sawing machine, material, operation, and workmanship, and may not be detected within short time. Conventionally, manufacturing yield is evaluated applying typical yield measure index method under the assumptions that the processes are stable and normal. To assess manufacturing yield for Gamma wafer sawing processes more accurately, we present a modified yield measure index method. Using the proposed method, the magnitudes of the undetected variance change, which are functions of the detection power of the $S^{2}$ chart, are incorporated into the evaluation of manufacturing yield. In addition, we demonstrate, mathematically, that the accommodation would not be affected by the scale parameter of Gamma distribution. For illustration purpose, a real-world case in a wafer sawing factory which is located on the Science-based Industrial Park in Hsinchu, Taiwan, is presented.
\end{abstract}

Index Terms - Cracking, gamma process, manufacturing yield, $S^{2}$ chart, variance change, wafer sawing.

\section{INTRODUCTION}

$\mathbf{R}$ APIDLY rising sales of portable devices cause the global market for small- and medium-sized thin film transistor liquid crystal display (TFT-LCD) panels to expand at its fastest pace in recent years. In the manufacturing technology of TFTLCD, driver integrated circuit (IC) is a critical device that is embedded with sophisticated circuits to drive panels. To satisfy the trend of modern portable devices applications on lighter and thinner requirements, narrow border designs on LCD panels have become more popular. Because of the narrow border design, space left for a LCD driver IC on current mechanism panel design is smaller, as depicted in Fig. 1. Consequently, dimensions of driver IC are shrunken. In the

Manuscript received May 6, 2010; revised December 11, 2010; accepted February 21, 2011. Date of current version August 12, 2011. Recommended for publication by Associate Editor S. J. Mason upon evaluation of reviewers' comments.

W.-L. Pearn, K.-B. Huang, and P.-L. Ku are with the Department of Industrial Engineering and Management, National Chiao Tung University, Hsinchu 300, Taiwan (e-mail: wlpearn@mail.nctu.edu.tw; treeup.iem95g@ nctu.edu.tw; s9633525.iem96g@g2.nctu.edu.tw).

Y.-T. Tai is with the Department of Information Management, Kainan University, Taoyuan 338, Taiwan (e-mail: yttai@mail.knu.edu.tw).

Color versions of one or more of the figures in this paper are available online at http://ieeexplore.ieee.org.

Digital Object Identifier 10.1109/TCPMT.2011.2134853 high-density LCD driver ICs, the operation of wafer sawing is essential and needs accurate yield assessment since understanding process and quantifying process performance are essential for any successful quality improvement initiative. To assess manufacturing yield for Gamma wafer sawing processes more precisely, we present a modified yield measure index method to accommodate undetected variance changes.

In the manufacturing process of driver ICs, the wafer sawing process is essential, which is a destructiveness process that uses diamond blade and sawing machine to separate dices. Fig. 2 presents the sawing process with a blade. In the wafer sawing process, cracking and chipping are two of the key failure modes for subsequent electronic packages [1]-[4]. Fig. 3 shows that chipping occurs when a wafer is sawed using a diamond blade. Notably, since the smaller space limits the dimensions of LCD driver IC due to narrow border design, particularly, on the width of driver ICs, the target of wafer sawing has been tightened and specified. However, some inevitable process variations and shifts regarding inadequate sawing may occur due to the limitations of sawing equipment. Therefore, it is essential to assess the manufacturing yield more accurately on wafer sawing process and further control the manufacturing processes.

As rapid advancement of the wafer sawing technology, customers require their products to be high quality with very low fraction of defectives. Classical methods [5]-[7] for measuring fraction of defectives become inapplicable for those high quality processes since any manufacturing sample of reasonable size likely contains no defective product items. In wafer sawing factories, control charts are commonly used to provide early warning for the shifts in the process mean and variance. Yield measure index is usually applied to measure the manufacturing yield. However, resulting from sawing machine, material and workmanship, some process variance changes may not be detected under the designated detection powers of control charts. Due to the undetected variance changes in the wafer sawing process, we will wrongly use the yield measure index. In fact, the actual value of yield measure index $\mathcal{C}_{p k}$ is decreased. However, conventional capability evaluating formulae are not adjusted to accommodate the undetected variance changes. Consequently, wafer sawing yield is overestimated. In this paper, we develop a correct adjusted yield assessment formula to obtain wafer sawing yield more accurately, as it could provide feedback to engineers on what actions need to take for manufacturing yield control and improvement.

In this paper, the manufacturing yields are assessed for Gamma wafer sawing processes incorporating variance 


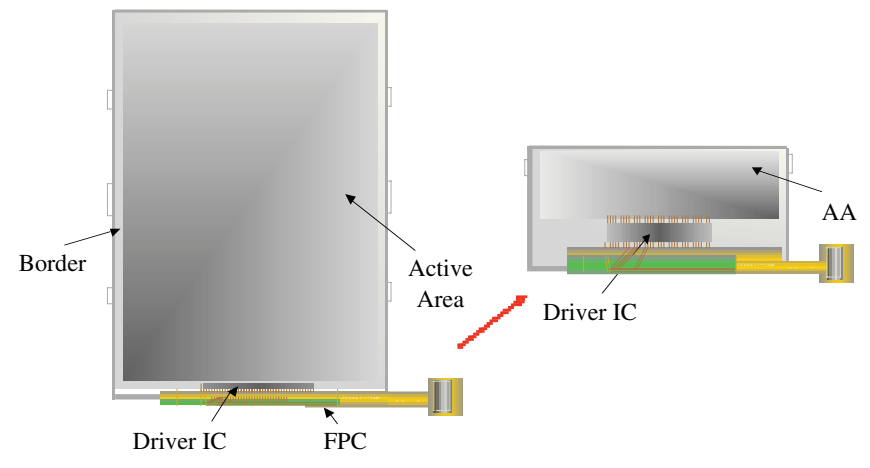

Fig. 1. Mechanism of narrow border.

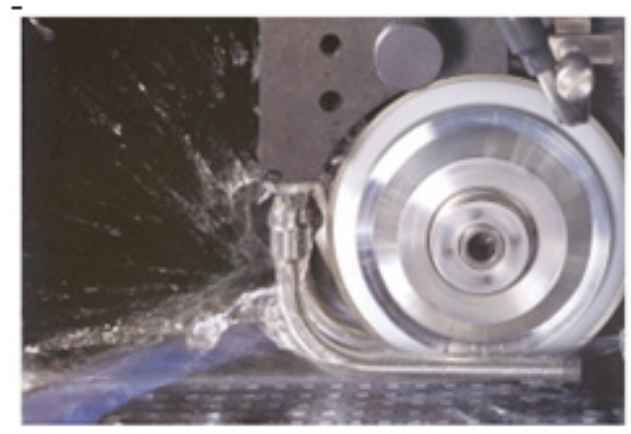

Fig. 2. Wafer sawing with a blade.

change conditions since some inadequate wafer sawing may occur and is very often skewed. In addition, Pyzdek [8] and Choi et al. [9] mentioned that the distributions of certain chemical processes or wafer production processes are also skewed. Gamma distribution (skewed), denoted as Gamma $(\alpha, \beta)$, with various values of $\alpha$ and $\beta$, covers a wide class of non-normal applications, including the manufacturing of semiconductor products, head or gimbal assembly for memory storage systems, and many others. This paper is organized as follows. Section II investigates manufacturing yield problem in the wafer sawing process. Section III shows the manufacturing yield assessment using a yield measure index method and presents the statistical properties of sample variance for Gamma wafer sawing processes. We provide a manufacturing yield assessment to accommodate undetected variance changes. To illustrate the applicability of the proposed manufacturing yield assessment, a real-world case taken from the manufacturing process in a wafer sawing factory is presented in Section IV. We also mathematically show that the accommodation of manufacturing yield assessment would not be affected by the scale parameter of a Gamma distribution. Finally, Section V provides the conclusions.

\section{Manufacturing Yield of The Wafer SAWING PROCESS}

In the manufacturing processes of LCD driver IC, there are four major manufacturing stages. At the first stage, ICs are designed by IC designers, and then are constructed on silicon wafers in wafer fabrications. At the second stage, the gold bumps are formed and mounted on wafers. At the third stage,

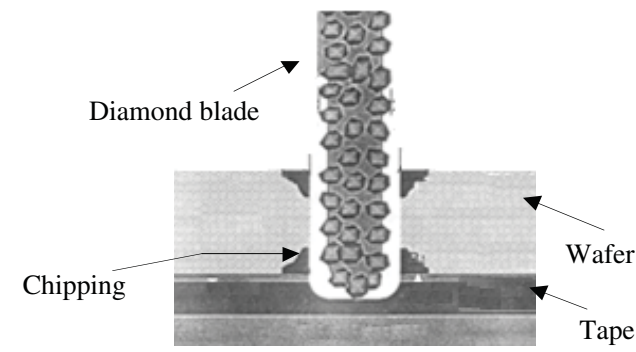

Fig. 3. Chipping occurs when dicing saw.

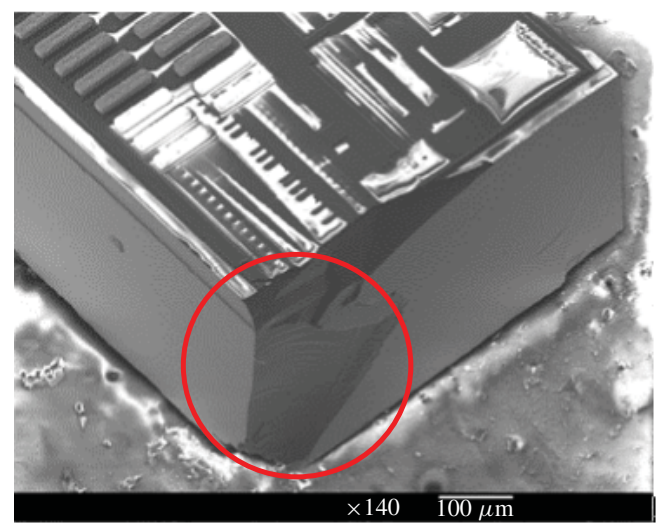

Fig. 4. Driver IC chipping $(\times 140)$.

chip probing test is performed. At the last stage, a processed and tested wafer is separated into individual IC using the wafer sawing process. Then, the LCD driver IC would mount onto glass substrate through chip on glass (COG) packaging. Wafer sawing process is the essential stage of the whole driver IC manufacturing processes. To fit the smaller dimension or space left for LCD driver IC on current mechanism panel design, which is depicted in Fig. 1 (see Section I), LCD driver ICs must be designed to be thinner and longer. Consequently, the IC design trend leads to the control of wafer sawing processes becoming more essential.

One of the key parameters of wafer sawing process control is sawing wastage. The sawing wastage affects the actual width of LCD driver IC in two ways. First, the less sawing wastage leads to the wider width of driver IC. The wider width of driver IC could not be acceptable for original panel design, which may be over the dimension of glass substrate. Moreover, the wider dimension of driver IC may also block original flexible printed circuit (FPC) design. It causes driver IC or FPC dropping from glass substrate, and certainly malfunction. Thinner width of driver IC is better for narrow border display panel design, but it suffers another problem. They are common IC cracking/chipping problems. Cracking/chipping in a driver IC could be regarded as sawing wastage. They may be the root cause of uneven topside or backside chipping, and break. Figs. 4 and 5 show the phenomena of chipping and cracking on driver ICs, respectively. Fig. 6 presents that the driver IC with severe cracking/chipping should be scrapped since the layer of metal has been destroyed.

Notably, IC cracking/chipping becomes more serious since the dimensions of driver IC are thinner and longer in current 


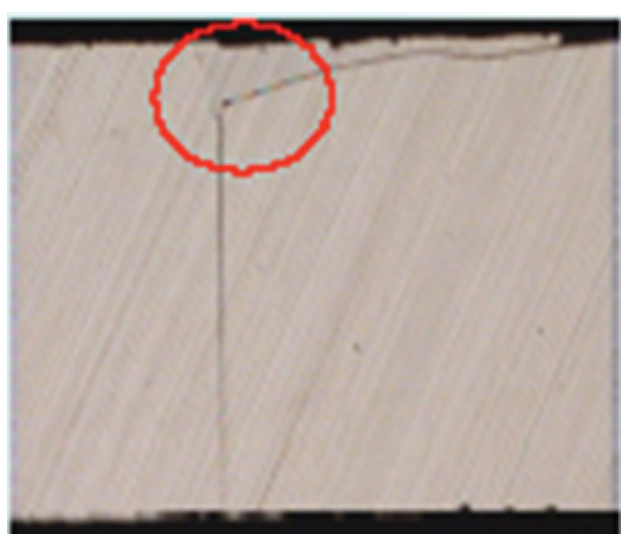

Fig. 5. Driver IC cracking.

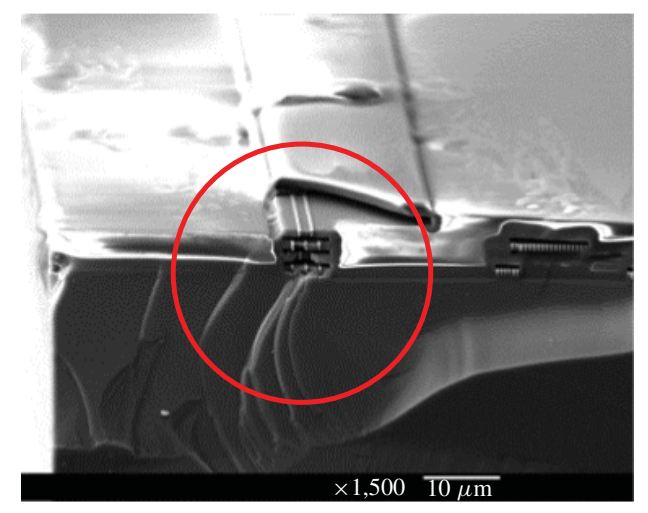

Fig. 6. Metal layer has been destroyed after scribing.

popular application of high definition display. For example, the dimensions of $700 \times 24000 \mu \mathrm{m}$ is a current chip size of a driver IC of wide video graphics array (WVGA) resolution $(480$ RGB $\times$ 864). Longer and thinner dimensions increase the difficulties of sawing process because the scribe lanes between the individual driver ICs is only around $80 \mu \mathrm{m}$ and the width of diamond blade is more than $20 \mu \mathrm{m}$. Narrow sawing lane will endanger the performance in the sawing process [10].

In the wafer sawing operation, there are some controllable variables (cutting speed, feeding speed, depth of cut and tool geometry) which determine relevant outputs (surface quality on kerf width, surface and straightness). Wafer sawing process is stable (i.e., in control) under statistical process control. However, some inevitable process variance changes which could arise from equipment, material and workmanship, and may not be detected within short time. In this paper, to circumvent the undetected variance changes causing the inaccurate manufacturing yield calculation, we present a modified yield measure index to avoid overestimating the manufacturing yield. It is an essential part of implementing any quality improvement program.

\section{Manufacturing Yield Assessment Using YIELD MEASURE INDEX}

Accurate assessment of manufacturing yield in TFT-LCD industry is essential. Due to high technology product requiring
TABLE I

CORRESPONDING PROCESS YIELD AND NCPPM FOR VARIOUS VALUES OF CAPABILITY INDEX

\begin{tabular}{|c|c|c|c|c|c|}
\hline $\mathcal{C}_{p k}$ & Yield & NCPPM & $\mathcal{C}_{p k}$ & Yield & NCPPM \\
\hline 0.9 & 0.993066052 & 6933.950 & 1.5 & 0.999993205 & 6.795 \\
\hline 1.0 & 0.997300204 & 2699.796 & 1.6 & 0.999998413 & 1.587 \\
\hline 1.1 & 0.999033152 & 966.848 & 1.67 & 0.999999456 & 0.544 \\
\hline 1.2 & 0.999681783 & 318.217 & 1.7 & 0.999999660 & 0.340 \\
\hline 1.3 & 0.999903807 & 96.193 & 1.8 & 0.999999933 & 0.067 \\
\hline 1.33 & 0.999933927 & 66.073 & 1.9 & 0.999999988 & 0.012 \\
\hline 1.4 & 0.999973309 & 26.692 & 2.0 & 0.999999998 & 0.002 \\
\hline
\end{tabular}

very low fraction of defectives, a capability index is employed to assess manufacturing yield in wafer sawing processes. Conventional yield measure index is applied to evaluate manufacturing yield under stable normal process [11], [12]. However, the wafer sawing process is a Gamma process with undetected variance changes due to the limitations of sawing equipments, materials and workmanship. Furthermore, some huge-damaged cracks or chipping may occur, the wafer sawing process is often skewed as Gamma process.

\section{A. Manufacturing Yield for Stable Normal Process}

In wafer sawing factories, yield measure index approach is used as an effective tool to assess manufacturing yield. This approach can measure the process departures from the target value and the magnitude of process variance. The index $\mathcal{C}_{p k}$ has been viewed as an yield-based index since it provides bounds of the process yield for a normally distributed process with a fixed value of $\mathcal{C}_{p k}$. The index proposed by Kane [13] and defined as $\min \{(U S L-\mu) / 3 \sigma,(\mu-L S L) / 3 \sigma\}$, where $U S L$ and $L S L$ are the upper and lower specification limits, respectively, $\mu$ is the process mean and $\sigma$ is the process standard deviation. For yield evaluation, one would statistically expect that the product's fractions of defectives, is no more than 2700 parts per million (ppm) while the value of $\mathcal{C}_{p k}$ is equal to 1 . At $\mathcal{C}_{p k}=1.33$, the defect rate drops to $66 \mathrm{ppm}$. To attain less than $0.544 \mathrm{ppm}$ defect rate, a $\mathcal{C}_{p k}$ level of 1.67 is required (see [11]). Table I presents various commonly used capability requirement and the corresponding overall process yield associated with non-conformities (NC) in parts per million (NCPPM).

\section{B. Manufacturing Yield for Gamma Process with Undetected Variance Changes}

Wafer sawing process is Gamma process with undetected variance changes owing to various limitations of equipment. The yield-based index $\mathcal{C}_{p k}$ is critical to the assumptions that the process is stable and normal. Bothe [14] considered dynamic mean shifts for normal processes. However, the wafer sawing process is not approximately normal. Hsu et al. [15] investigated mean shifts for Gamma process under dynamic manufacturing environment. In wafer sawing factories, the variance changes may exist but undetected in the shop floor. 
TABLE II

Detection Power of VArious Values of $\alpha$ From Gamma Distributions with $n=10$

\begin{tabular}{|c|c|c|c|c|c|c|c|c|c|c|c|}
\hline \multirow{2}{*}{ Change $\sigma$} & \multicolumn{10}{|c|}{ Distribution of Gamma $(\alpha, 1)$} \\
\cline { 2 - 13 } & $\alpha=0.5$ & $\alpha=1$ & $\alpha=2$ & $\alpha=3$ & $\alpha=4$ & $\alpha=5$ & $\alpha=6$ & $\alpha=7$ & $\alpha=8$ & $\alpha=9$ & $\alpha=10$ \\
\hline 1 & 0.0026 & 0.0026 & 0.0026 & 0.0029 & 0.0028 & 0.0027 & 0.0027 & 0.0027 & 0.0026 & 0.0027 & 0.0027 \\
\hline 1.5 & 0.0506 & 0.0542 & 0.0684 & 0.0801 & 0.0914 & 0.1000 & 0.1078 & 0.1126 & 0.1184 & 0.1241 & 0.1284 \\
\hline 2 & 0.1520 & 0.1691 & 0.2230 & 0.2688 & 0.3077 & 0.3400 & 0.3665 & 0.3883 & 0.4102 & 0.4250 & 0.4387 \\
\hline 2.5 & 0.2608 & 0.2766 & 0.3522 & 0.4213 & 0.4778 & 0.5246 & 0.5631 & 0.5970 & 0.6196 & 0.6440 & 0.6645 \\
\hline 3 & 0.3561 & 0.3604 & 0.4312 & 0.5070 & 0.5737 & 0.6264 & 0.6713 & 0.7050 & 0.7360 & 0.7560 & 0.7769 \\
\hline 3.5 & 0.4399 & 0.4280 & 0.4826 & 0.5561 & 0.6239 & 0.6780 & 0.7221 & 0.7565 & 0.7856 & 0.8105 & 0.8321 \\
\hline 4 & 0.5092 & 0.4849 & 0.5223 & 0.5830 & 0.6475 & 0.7006 & 0.7467 & 0.7812 & 0.8110 & 0.8364 & 0.8554 \\
\hline 4.5 & 0.5711 & 0.5325 & 0.5521 & 0.6039 & 0.6606 & 0.7102 & 0.7545 & 0.7885 & 0.8202 & 0.8436 & 0.8645 \\
\hline 5 & 0.6217 & 0.5783 & 0.5784 & 0.6196 & 0.6691 & 0.7157 & 0.7566 & 0.7894 & 0.8203 & 0.8456 & 0.8677 \\
\hline
\end{tabular}

TABLE III

Detection Power of Various Values of From Gamma Distributions with $n=15$

\begin{tabular}{|c|c|c|c|c|c|c|c|c|c|c|c|}
\hline \multirow{2}{*}{ Change $\sigma$} & \multicolumn{10}{|c|}{ Distribution of Gamma $(\alpha, 1)$} \\
\cline { 2 - 14 } & $\alpha=0.5$ & $\alpha=1$ & $\alpha=2$ & $\alpha=3$ & $\alpha=4$ & $\alpha=5$ & $\alpha=6$ & $\alpha=7$ & $\alpha=8$ & $\alpha=9$ & $\alpha=10$ \\
\hline 1 & 0.0027 & 0.0028 & 0.0027 & 0.0027 & 0.0027 & 0.0027 & 0.0027 & 0.0027 & 0.0027 & 0.0026 & 0.0027 \\
\hline 1.5 & 0.0569 & 0.0708 & 0.0985 & 0.1183 & 0.1368 & 0.1515 & 0.1634 & 0.1756 & 0.1836 & 0.1925 & 0.1978 \\
\hline 2 & 0.1701 & 0.2199 & 0.3136 & 0.3817 & 0.4401 & 0.4885 & 0.5266 & 0.5553 & 0.5828 & 0.6006 & 0.6204 \\
\hline 2.5 & 0.2775 & 0.3361 & 0.4685 & 0.5644 & 0.6373 & 0.6911 & 0.7346 & 0.7703 & 0.7942 & 0.8140 & 0.8353 \\
\hline 3 & 0.3667 & 0.4181 & 0.5503 & 0.6523 & 0.7273 & 0.7844 & 0.8232 & 0.8541 & 0.8785 & 0.8960 & 0.9111 \\
\hline 3.5 & 0.4408 & 0.4773 & 0.5943 & 0.6937 & 0.7683 & 0.8218 & 0.8599 & 0.8887 & 0.9122 & 0.9276 & 0.9401 \\
\hline 4 & 0.5045 & 0.5228 & 0.6204 & 0.7133 & 0.7834 & 0.8356 & 0.8737 & 0.9013 & 0.9227 & 0.9388 & 0.9513 \\
\hline 4.5 & 0.5591 & 0.5605 & 0.6388 & 0.7237 & 0.7886 & 0.8381 & 0.8772 & 0.9045 & 0.9244 & 0.9423 & 0.9540 \\
\hline 5 & 0.6060 & 0.5938 & 0.6537 & 0.7260 & 0.7879 & 0.8365 & 0.8749 & 0.9031 & 0.9248 & 0.9410 & 0.9533 \\
\hline
\end{tabular}

Aforementioned undetected variance changes indeed overestimate the manufacturing yield and mislead the practitioners to take improper actions. To control the manufacturing stability under dynamic manufacturing environment, the $S^{2}$ chart is employed to monitor the process variance. Consequently, the optimal and appropriate approach is to incorporate the undetected variance changes for manufacturing yield assessment. We investigate the Gamma distribution to realize the effect on the detection power of the control chart. Observations from the Gamma distribution are non-negative. The Gamma distribution can be denoted as Gamma $(\alpha, \beta)$ with shape parameter $\alpha$ and scale parameter $\beta$ random variable is said to have a Gamma distribution with parameters $(\alpha, \beta) \alpha>0, \beta>0$, if its density function is given by

$$
f(x)=\frac{1}{\Gamma(\alpha) \beta^{\alpha}} x^{\alpha-1} \exp ^{\frac{-x}{\beta}} \quad x>0, \alpha>0, \beta>0
$$

and the mean and variance are given by $\mu=\alpha \beta$ and $\sigma^{2}=\alpha \beta^{2}$, respectively [16]. Since the manufacturing yield should be measured accurately under Gamma processes, an accommodation for the undetected variance changes is referred to as $A S_{50}$ which would be the marginal size of the undetected variance change we should accommodate based on designated detection power is $0.5 . A S_{50}$ is a function of the detection power of the $S^{2}$ chart.

Control charts can quickly detect the occurrence of variability or process shifts so that proper corrective actions may be undertaken before defective units are manufactured [11]. In this subsection, since the effects on the manufacturing yield assessment for Gamma wafer sawing process is investigated, the $S^{2}$ chart is a common tool to monitor process variability and can help us quickly determine whether the process is stable or not.

1) Stability Control Using $S^{2}$ Chart for Gamma Process: To enhance the stability of variance, the $S^{2}$ chart is usually applied. However, there is a critical assumption, which is that the process characteristics must follow normal, that should be satisfied. Since the wafer sawing process is Gamma process, we would need to replace the traditional $U C L$ and $L C L$ with $\left(\bar{S}^{2} / n-1\right) \chi_{\alpha / 2, n-1}^{2}$ and $\left(\bar{S}^{2} / n-1\right) \chi_{1-(\alpha / 2), n-1}^{2}$, respectively. The new ones represent quantiles of the cumulative distribution function from different parameters of Gamma $(\alpha, \beta)$.

However, the UCL and LCL are rather difficult to obtain the explicit formulas for Gamma processes. In this paper, the Monte-Carlo simulation method is performed to investigate the behavior of sampling distribution of variance for Gamma processes and is used to determine the estimated UCL and LCL. Steps of the Monte-Carlo algorithm which determine the control limits of $S^{2}$ chart are summarized as follows.

Step 1: Generate random sample $X_{1}, X_{2}, \ldots, X_{n}$ from Gamma distribution $G\left(\alpha_{0}, \beta_{0}\right) k$ times independently, simulating $X_{1}^{(i)}, \ldots, X_{n}^{(i)} \sim G\left(\alpha_{0}, \beta_{0}\right)$, $i=1,2, \ldots, k$. 
TABLE IV

Detection Power of Various Values Of $\alpha$ From Gamma Distributions with $n=30$

\begin{tabular}{|c|c|c|c|c|c|c|c|c|c|c|c|}
\hline \multirow{2}{*}{ Change $\sigma$} & \multicolumn{10}{|c|}{ Distribution of Gamma $(\alpha, 1)$} \\
\cline { 2 - 13 } & $\alpha=0.5$ & $\alpha=1$ & $\alpha=2$ & $\alpha=3$ & $\alpha=4$ & $\alpha=5$ & $\alpha=6$ & $\alpha=7$ & $\alpha=8$ & $\alpha=9$ & $\alpha=10$ \\
\hline 1 & 0.0028 & 0.0027 & 0.0026 & 0.0027 & 0.0029 & 0.0028 & 0.0028 & 0.0026 & 0.0027 & 0.0028 & 0.0028 \\
\hline 1.5 & 0.0821 & 0.1259 & 0.1921 & 0.2444 & 0.2878 & 0.3305 & 0.3566 & 0.3818 & 0.4140 & 0.4283 & 0.4385 \\
\hline 2 & 0.2458 & 0.3693 & 0.5560 & 0.6690 & 0.7516 & 0.8022 & 0.8335 & 0.8663 & 0.8866 & 0.9035 & 0.9137 \\
\hline 2.5 & 0.3652 & 0.5213 & 0.7216 & 0.8337 & 0.8965 & 0.9340 & 0.9531 & 0.9663 & 0.9764 & 0.9824 & 0.9863 \\
\hline 3 & 0.4483 & 0.5979 & 0.7918 & 0.8881 & 0.9379 & 0.9635 & 0.9785 & 0.9865 & 0.9918 & 0.9946 & 0.9960 \\
\hline 3.5 & 0.4979 & 0.6373 & 0.8188 & 0.9055 & 0.9500 & 0.9734 & 0.9847 & 0.9913 & 0.9947 & 0.9969 & 0.9981 \\
\hline 4 & 0.5462 & 0.6583 & 0.8261 & 0.9115 & 0.9545 & 0.9751 & 0.9861 & 0.9923 & 0.9955 & 0.9975 & 0.9984 \\
\hline 4.5 & 0.5854 & 0.6735 & 0.8256 & 0.9081 & 0.9523 & 0.9741 & 0.9859 & 0.9923 & 0.9955 & 0.9973 & 0.9985 \\
\hline 5 & 0.6181 & 0.6878 & 0.8223 & 0.9041 & 0.9483 & 0.9710 & 0.9838 & 0.9910 & 0.9948 & 0.9969 & 0.9983 \\
\hline
\end{tabular}

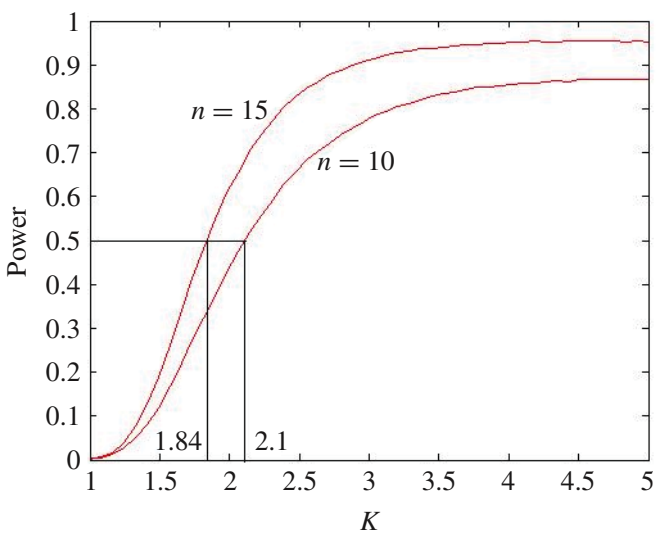

(a)

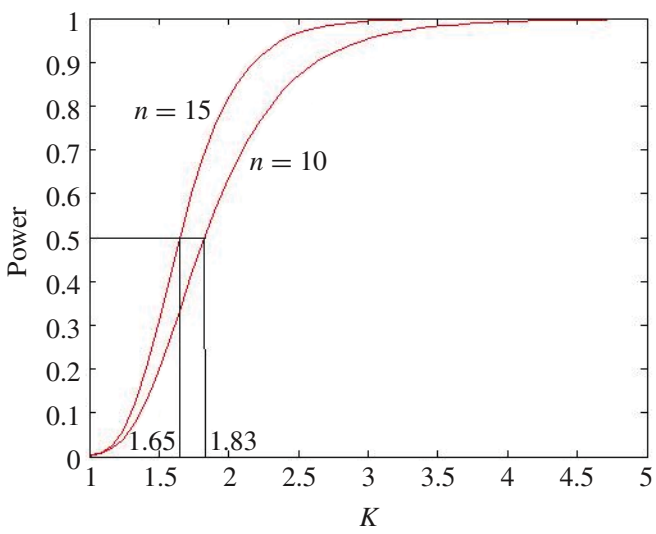

(b)

Fig. 7. Power curves with: (a) $\alpha=10$ and (b) $\alpha=100$ for various subgroup sizes 10,15 .

Step 2: Calculate $S^{2(i)}=\sum_{j=1}^{n}\left(X_{j}^{(i)}-\bar{X}^{(i)}\right)^{2} / n-1$, where $\bar{X}^{(i)}=\sum_{j=1}^{n} X_{j}^{(i)} / n, \quad i=1,2, \ldots, k$.

Step 3: Arrange the simulated observations $S^{2(1)}, S^{2(2)}$, $\ldots, S^{2(k)}$ in an increasing order. Denote $S_{(i)}^{2}$ as the $i^{\text {th }}$ order statistic for $S^{2(1)}, S^{2(2)}, \ldots, S^{2(k)}$, hence, we have $S_{(1)}^{2}<S_{(2)}^{2}<\cdots<S_{(k)}^{2}$.

Step 4: Calculate the $[100 \times(1-\alpha)]^{\text {th }}$ sample percentile $S_{([k(1-\alpha)])}^{2}$, where $[k(1-\alpha)]$ is the largest integer less than or equal to $k(1-\alpha)$. Then, $S_{([k(1-\alpha)])}^{2}$ is a Monte-Carlo estimator for $F_{s^{2}}^{-1}(1-\alpha)$.

Utilizing the $U C L$ and $L C L$ obtained by the Monte-Carlo approach, the power of $S^{2}$ chart for Gamma distributed data is derived. Since the Type II error $\beta$ is

$$
\begin{aligned}
\beta & =P\left(L C L \leq S^{2} \leq U C L \mid \sigma_{1}=K \sigma_{0}\right) \\
& =P\left(F_{0.135} \leq S^{2} \leq F_{99.865} \mid \sigma_{1}=K \sigma_{0}\right) \\
& =G_{S^{2}}\left(F_{99.865}\right)-G_{S^{2}}\left(F_{0.135}\right)
\end{aligned}
$$

where $1-\beta$ is the detection power of the process, $G_{S^{2}}(\cdot)$ is the empirical cumulative distribution function of sample variance from Gamma distribution with that variance has changed and $\sigma_{1}$ is the new standard deviation after the variance change ( $\sigma_{0}$ is the standard deviation of the original process). The two control limits, $L C L$ and $U C L$, are calculated as
$F_{0.135}$ and $F_{99.865}$, respectively, where $F_{\delta}$ represents the $\delta$ th percentile.

Tables II-IV present the detection powers of the Gamma distribution with the parameter $\alpha$ varied from $0.5,1,2, \ldots, 10$, while fixing $\beta=1$ and subgroup sizes are set to be 10,15 , and 30 , respectively. The first column on the left side is the magnitude of variance change. It can be seen from Tables II-IV that the detection power increases as $\alpha$ increases.

2) Manufacturing Yield Assessment: In Gamma wafer sawing manufacturing environment, magnitudes of variance change may be undetected. To measure the manufacturing yield more accurately, we incorporate $A S_{50}$ into the yield assessment formula to accommodate yield measure index. The reason which we set the $A S_{50}$ (the detection power is 0.5 ) is that we apply the average run length (ARL) $=2$ since it is the commonly used run number for stopping the manufacturing line when the out of control signal is detected. As ARL $=2$, the detection power is $1 / \mathrm{ARL}=(1 / 2)=0.5$. Furthermore, we develop a computation using a commercial package to compute the accommodation of variance change $\left(A S_{50}\right)$. We assume that Gamma process with various values of $\alpha=$ 0.5 and $1,2,3, \ldots, 10, \beta=1$ and $n=10,11,12, \ldots, 30$. For example, we set $\alpha=7$ and $n=15$, the magnitude of accommodation of variance change is 1.92 (see Table V). It also reveals from Table $\mathrm{V}$ that the value of $A S_{50}$ get closer to the accommodation under normal population as $\alpha$ increases. 
TABLE V

$A S_{50}$ VAlues For SeVERAL Subgroup Sizes $n$ AND VARious $\alpha$ VALUES

\begin{tabular}{|l|c|c|c|c|c|c|c|c|c|c|c|}
\hline$n$ & 0.5 & 1 & 2 & 3 & 4 & 5 & 6 & 7 & 8 & 9 & 10 \\
\hline 10 & 3.91 & 4.15 & 3.70 & 2.93 & 2.59 & 2.41 & 2.30 & 2.23 & 2.17 & 2.14 & 2.10 \\
\hline 11 & 3.93 & 4.09 & 3.42 & 2.74 & 2.44 & 2.31 & 2.21 & 2.15 & 2.10 & 2.07 & 2.03 \\
\hline 12 & 3.95 & 4.03 & 3.15 & 2.58 & 2.35 & 2.21 & 2.13 & 2.07 & 2.03 & 2.00 & 1.97 \\
\hline 13 & 3.98 & 3.92 & 2.97 & 2.47 & 2.24 & 2.14 & 2.06 & 2.01 & 1.97 & 1.95 & 1.92 \\
\hline 14 & 3.96 & 3.86 & 2.81 & 2.36 & 2.18 & 2.07 & 2.01 & 1.96 & 1.93 & 1.89 & 1.87 \\
\hline 15 & 3.96 & 3.74 & 2.67 & 2.28 & 2.11 & 2.02 & 1.96 & 1.92 & 1.88 & 1.86 & 1.84 \\
\hline 16 & 3.96 & 3.64 & 2.55 & 2.20 & 2.06 & 1.98 & 1.92 & 1.88 & 1.84 & 1.82 & 1.80 \\
\hline 17 & 3.96 & 3.53 & 2.46 & 2.15 & 2.01 & 1.93 & 1.88 & 1.84 & 1.81 & 1.79 & 1.77 \\
\hline 18 & 3.94 & 3.40 & 2.39 & 2.10 & 1.97 & 1.90 & 1.84 & 1.81 & 1.78 & 1.75 & 1.74 \\
\hline 19 & 3.92 & 3.26 & 2.31 & 2.05 & 1.93 & 1.86 & 1.81 & 1.78 & 1.75 & 1.73 & 1.71 \\
\hline 20 & 3.9 & 3.16 & 2.26 & 2.02 & 1.90 & 1.83 & 1.79 & 1.75 & 1.72 & 1.71 & 1.69 \\
\hline 21 & 3.96 & 3.03 & 2.22 & 1.98 & 1.86 & 1.81 & 1.76 & 1.73 & 1.71 & 1.68 & 1.68 \\
\hline 22 & 3.86 & 2.98 & 2.18 & 1.95 & 1.83 & 1.79 & 1.74 & 1.70 & 1.68 & 1.66 & 1.65 \\
\hline 23 & 3.77 & 2.82 & 2.12 & 1.93 & 1.82 & 1.76 & 1.72 & 1.70 & 1.66 & 1.65 & 1.62 \\
\hline 24 & 3.80 & 2.76 & 2.08 & 1.89 & 1.80 & 1.73 & 1.71 & 1.68 & 1.66 & 1.63 & 1.63 \\
\hline 25 & 3.76 & 2.68 & 2.05 & 1.87 & 1.76 & 1.71 & 1.68 & 1.66 & 1.63 & 1.63 & 1.59 \\
\hline 26 & 3.71 & 2.66 & 2.02 & 1.85 & 1.75 & 1.70 & 1.67 & 1.64 & 1.62 & 1.59 & 1.58 \\
\hline 27 & 3.61 & 2.63 & 1.99 & 1.83 & 1.72 & 1.70 & 1.65 & 1.62 & 1.60 & 1.59 & 1.58 \\
\hline 28 & 3.59 & 2.52 & 1.99 & 1.81 & 1.71 & 1.67 & 1.63 & 1.61 & 1.58 & 1.58 & 1.56 \\
\hline 29 & 3.46 & 2.45 & 1.95 & 1.78 & 1.69 & 1.65 & 1.61 & 1.60 & 1.58 & 1.57 & 1.55 \\
\hline 30 & 3.50 & 2.40 & 1.91 & 1.76 & 1.68 & 1.63 & 1.61 & 1.58 & 1.56 & 1.56 & 1.54 \\
\hline
\end{tabular}

It is reasonable since the corresponding distribution get closer to the standard normal distribution as $\alpha$ increases. However, when $\alpha$ is small (heavily skewed with long tail), the required accommodation in the yield measure index formula is much greater than those for normal processes. Utilizing the modified process capability formula, the practitioners can assess the process capability more accurately.

Fig. 7 presents the power curves. These lines on the graph depict the probabilities of detecting a change in $\sigma$ for several given subgroup sizes. For small changes in $\sigma$, all curves are close to zero. From Fig. 7(a) and (b), we can find that the power of the chart with all the curves leveling off close to $100 \%$ as the magnitude of variance change exceeds $4 \sigma$. The horizontal line drawn in Fig. 7(a) shows that there is a 50\% chance of not catch a $1.84 \sigma$ change in variance when $n$ is 15 , and missing the catch of $2.1 \sigma$ change when $n$ is 10 . Notably, variance change ranging from 0 up to $\mathcal{A S}_{50} \sigma$ is undetected using the $S^{2}$ chart. Consequently, the optimal approach to accommodate any variance change that is no greater than $\mathcal{A S}_{50} \sigma$. The modified capability formula can be expressed as follows. The definition for $C_{p u}$ and $C_{p l}$ are shown as follows:

$$
C_{p u}=\frac{U S L-\text { median }}{\text { (upper } 0.135 \% \text { point })- \text { median }}=\frac{U S L-M}{F_{99.865}-M}
$$

and

$$
C_{p l}=\frac{\text { median }-L S L}{\text { median }-(\text { lower } 0.135 \% \text { point })}=\frac{M-L S L}{M-F_{0.135}} .
$$

The index $\mathcal{C}_{p k}$ would be obtained as the minimum of $C_{p u}$ and $C_{p l}$, namely

$$
C_{p k}=\min \left\{C_{p u}, C_{p l}\right\}=\left\{\frac{U S L-M}{F_{99.865}-M}, \frac{M-L S L}{M-F_{0.135}}\right\}
$$

TABLE VI

VALUES OF SKEWNESS AND KURTOSIS FOR VARIOUS GAMMA DISTRIBUTIONS

\begin{tabular}{|c|l|l|}
\hline Distribution & Skewness & Kurtosis \\
\hline$N(0,1)$ & 0 & 3 \\
\hline Gamma $(10,1)$ & 0.6324 & 3.6 \\
\hline Gamma $(8,1)$ & 0.7071 & 3.75 \\
\hline Gamma $(6,1)$ & 0.8164 & 4 \\
\hline Gamma $(4,1)$ & 1 & 4.5 \\
\hline Gamma $(2,1)$ & 1.4142 & 6 \\
\hline Gamma $(1,1)$ & 2 & 9 \\
\hline
\end{tabular}

where $F_{\delta}$ is the $\delta$ th percentile point and $M$ is the median of the Gamma process.

3) Skewness and Kurtosis of Gamma Process: To further investigate how Gamma distribution is different from the standard normal distribution in terms of skewness and kurtosis, Table VI presents the values of skewness and kurtosis of the Gamma distributions. The skewness and kurtosis of Gamma $(\alpha, 1)$ are $2 / \sqrt{\alpha}$ and $6 / \alpha+3$, respectively. In Table VI, we find that the corresponding values of skewness and kurtosis will become large and far away from the values of the standard normal distribution when $\alpha$ decreases. In addition, from the formula of skewness and kurtosis, we can find that $\beta$ does not influence on skewness and kurtosis. No matter how we change the values of $\beta$, skewness and kurtosis will be no difference.

Fig. 8 presents several Gamma distributions along with normal distributions having the same mean and variance. In this investigation, we let $\alpha=1,2,4,6,8$, and 10 , while fixing $\beta=1$. It follows from Fig. 8 that as $\alpha$ increases, the Gamma distribution appears more nearly normal distribution. 


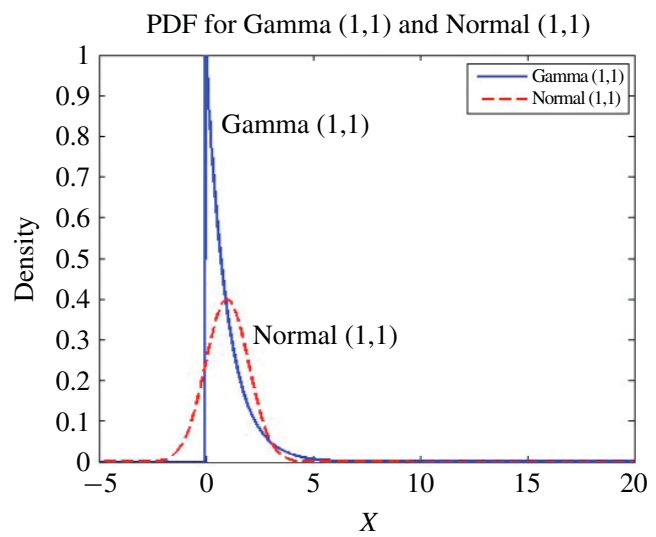

(a)

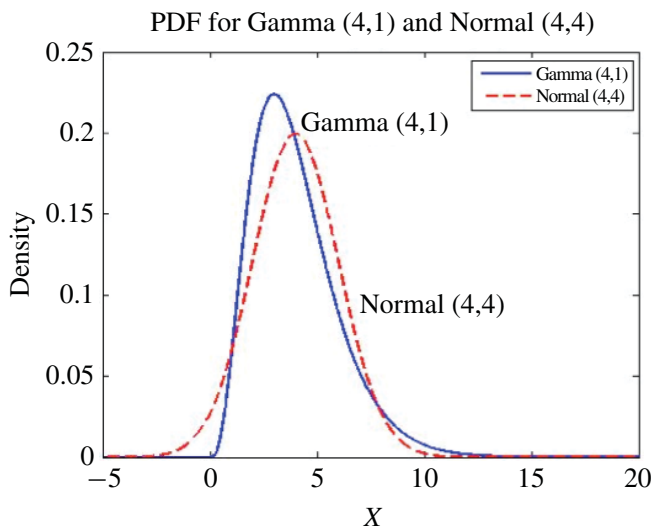

(c)

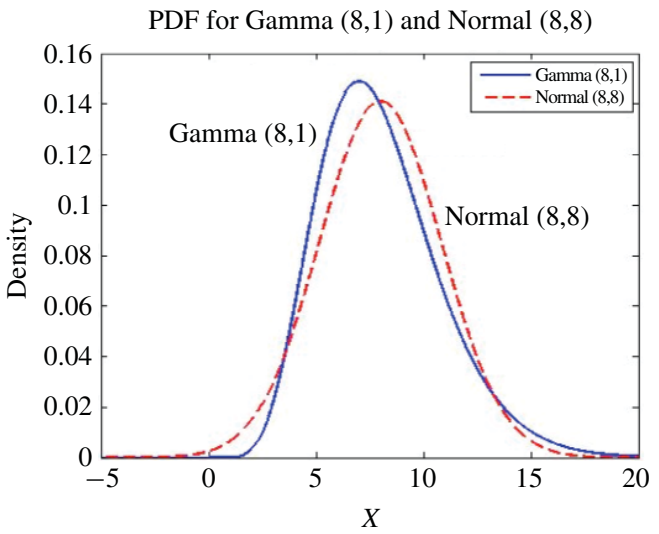

(e)

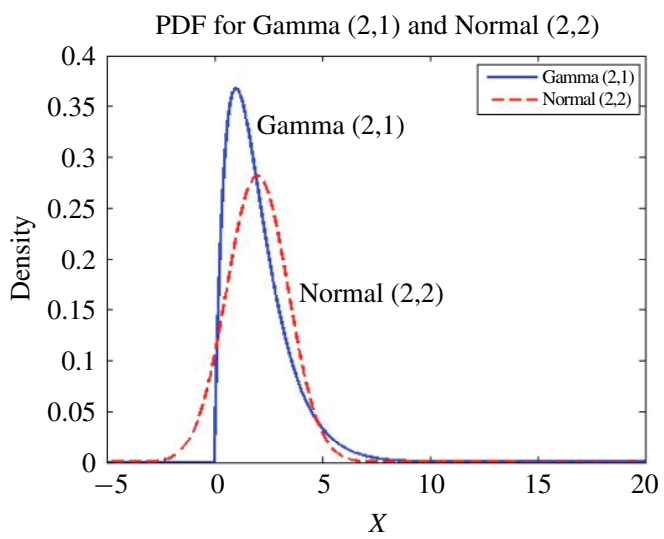

(b)

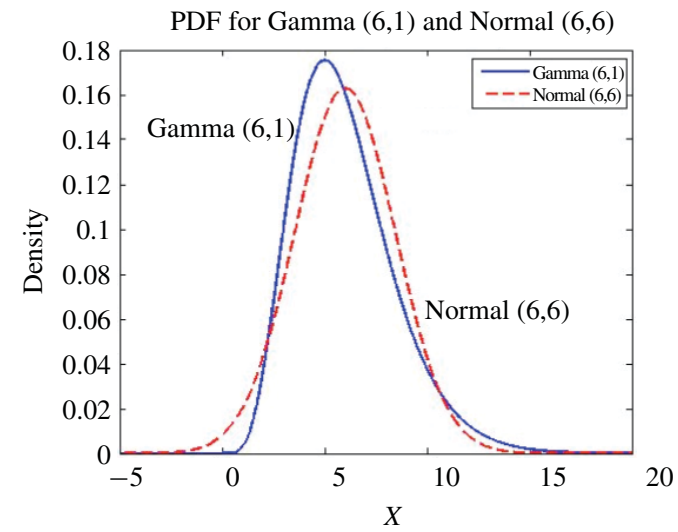

(d)

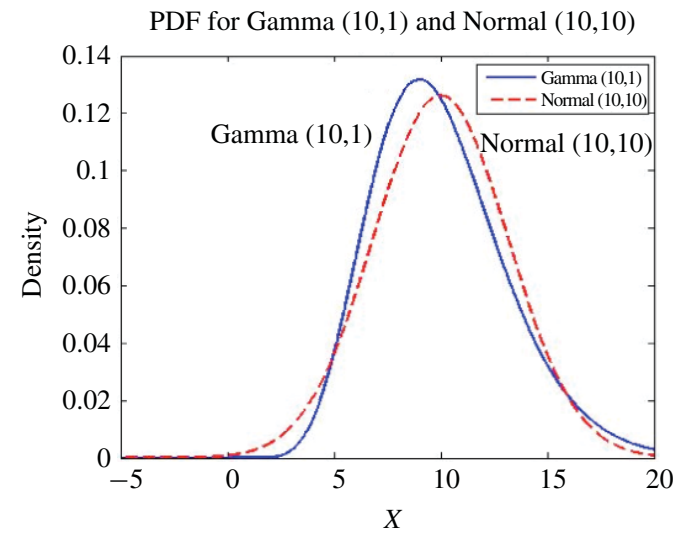

(f)

Fig. 8. Probability density functions for Gamma and Normal distributions with identical mean and various variances. (a) Gamma (1, 1) and $N(1,1)$. (b) Gamma $(2,1)$ and $N(2,2)$. (c) Gamma $(4,1)$ and $N(4,4)$. (d) Gamma $(6,1)$ and $N(6,6)$. (e) Gamma (8, 1) and $N(8,8)$. (f) Gamma $(10,1)$ and $N(10,10)$.

So we can infer that the sampling distribution of variance from Gamma distribution appears more nearly the sampling distribution of variance from normal population when $\alpha$ is large. As discussed above, we obtain some insights regarding the effects on the capability assessment when the process output follows non-normal distribution with process variance changes.

\section{Manufacturing Yield Assessment For Wafer SAWING PROCESSES}

To illustrate how to assess the manufacturing yield with undetected variance change conditions for Gamma wafer sawing processes, we consider the following case taken from a wafer sawing factory located on the Science-Based Industrial Park, Taiwan. For the case investigated, the product type of P351TR02, which belongs to WVGA resolution (480 RGB $\times$ 864) product series with 3.51-in panel-size for small-sized portable devices is considered. It is noted that the parameter, sawing wastage, is an essential specification for the wafer sawing process. We randomly collect the inspection data from the shop floor. Table VII presents the 100 measurement data of the product type P351TR02. The upper and lower specification limits of the sawing wastage for the particular model we investigated is usually set to 80 and $20 \mu \mathrm{m}$, respectively. 
TABLE VII

100 Observations Are Collected From The Historical Data

\begin{tabular}{|l|l|l|l|l|l|l|l|l|l|}
\hline 43.32 & 44.93 & 49.01 & 39.51 & 38.48 & 37.83 & 61.32 & 37.69 & 57.53 & 43.14 \\
\hline 49.98 & 42.85 & 45.36 & 44.64 & 37.93 & 41.55 & 41.86 & 37.49 & 54.30 & 58.30 \\
\hline 53.82 & 45.10 & 46.23 & 42.79 & 43.62 & 38.39 & 49.56 & 48.17 & 44.33 & 52.76 \\
\hline 54.93 & 45.37 & 42.17 & 54.16 & 44.30 & 49.89 & 38.70 & 46.71 & 39.10 & 52.62 \\
\hline 43.78 & 38.77 & 46.83 & 47.48 & 46.45 & 43.88 & 41.80 & 51.53 & 55.45 & 52.73 \\
\hline 46.13 & 43.71 & 60.85 & 46.45 & 42.24 & 43.81 & 43.61 & 41.31 & 40.02 & 44.07 \\
\hline 50.93 & 53.78 & 40.61 & 41.72 & 46.73 & 51.52 & 50.40 & 37.79 & 42.06 & 36.07 \\
\hline 43.98 & 53.10 & 42.51 & 49.34 & 50.54 & 51.42 & 52.11 & 37.95 & 47.74 & 36.65 \\
\hline 45.94 & 45.54 & 53.21 & 58.84 & 39.82 & 48.67 & 37.81 & 46.62 & 48.34 & 50.60 \\
\hline 40.35 & 50.56 & 45.48 & 38.51 & 47.38 & 37.19 & 46.98 & 37.34 & 50.15 & 39.24 \\
\hline
\end{tabular}

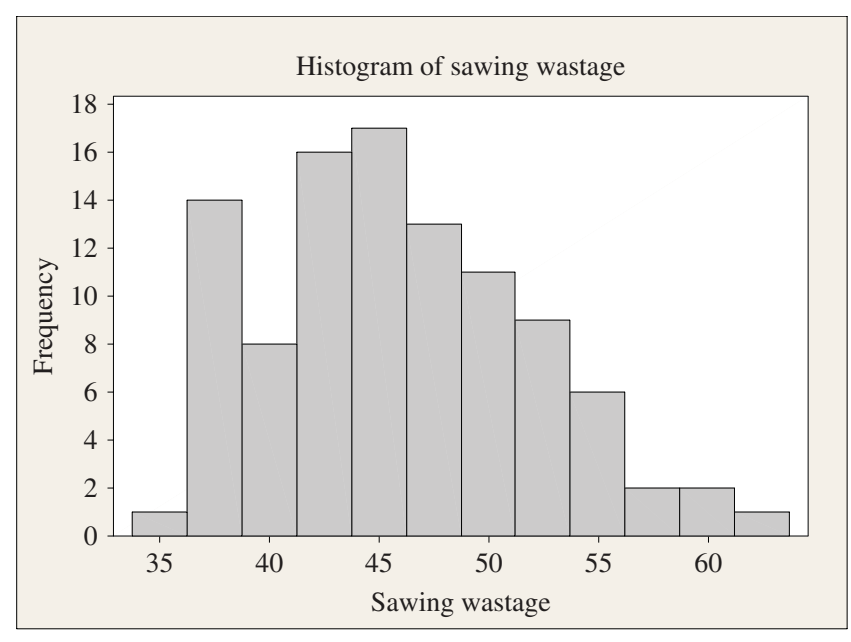

Fig. 9. Histogram plot of the historical data.

The ' $\mu \mathrm{m}$ ' is used as the unit for the specification of sawing wastage.

From Fig. 9, it is evident to conclude that the data collected from the factory are not normal. By the goodness-of-fit test, the historical data indicates that the process approximates to be distributed as Gamma ( $p$-value is equal to 0.692 using the Chi-squared test). The parameters $\alpha$ and $\beta$ of Gamma process could be obtained from historical data utilizing method of moments, giving $\hat{\alpha}=59.446$ and $\hat{\beta}=0.771$. We can apply the interpolation method and use following equations to obtain the three percentiles:

$$
\begin{aligned}
\hat{F}_{99.865}= & X_{\left(R_{1}\right)}+\left(\left[\frac{(99.865) n+0.135}{100}\right]-R_{1}\right) \\
& \times\left(X_{\left(R_{1}+1\right)}-X_{\left(R_{1}\right)}\right) \\
\hat{F}_{0.135}= & X_{\left(R_{2}\right)}+\left(\left[\frac{(0.135) n+99.865}{100}\right]-R_{2}\right) \\
& \times\left(X_{\left(R_{2}+1\right)}-X_{\left(R_{2}\right)}\right) \\
\hat{M}= & X_{\left(R_{3}\right)}+\left(\left[\frac{n+1}{2}\right]-R_{3}\right) \\
& \times\left(X_{\left(R_{3}+1\right)}-X_{\left(R_{3}\right)}\right)
\end{aligned}
$$

where $R_{1}=[(99.865 n+0.135) / 100], R_{2}=[(0.135 n+$ $99.865) / 100]$, and $R_{3}=[(n+1) / 2]$. In this setting, the notation $[R]$ is defined as the greatest integer less than or equal to the number $R$, and $x_{(i)}$ is defined as the $i$ th order statistic. Therefore, the three values can be obtained as follows:

$$
\hat{F}_{0.135}=36.148, \quad \hat{M}=45.365 \text {, and } \hat{F}_{99.865}=61.257 \text {. }
$$

Therefore, the modified $\mathcal{C}_{p k}$ index can be obtained in the following:

$$
\begin{aligned}
& \hat{C}_{p k} \\
& =\min \left\{\frac{U S L-\text { median }}{A S_{50}\left(\hat{F}_{99.865}-\text { median }\right)}, \frac{\text { median }-L S L}{A S_{50}\left(\text { median }-\hat{F}_{0.135}\right)}\right\} \\
& =\min \left\{\frac{80-45.365}{1.562(61.257-45.365)}, \frac{45.365-20}{1.562(45.365-36.148)}\right\} \\
& =1.395
\end{aligned}
$$

with the value of $A S_{50}$ as 1.562 when subgroup size $n$ is set to 20. Compared it to the value of following index $\hat{C}_{p k}=\min \left\{(U S L-\right.$ median $) /\left(\hat{F}_{99.865}-\right.$ median $),($ median $L S L) /\left(\right.$ median $\left.\left.-\hat{F}_{0.135}\right)\right\}=\min \{(80-45.365) /(61.257-$ $45.365),(45.365-20) /(45.365-36.148)\}=2.179$, which is evaluated by a conventional yield index investigation (the change of process variance is not considered). We can find that the value of the modified $\mathcal{C}_{p k}$ is much smaller. This result indicates that if the process variance changes are not detected then unadjusted $\mathcal{C}_{p k}$ would overestimate the manufacturing yield which is undesirable. Our accommodation considers undetected variance changes; therefore, industrial practitioners would make reliable decisions on controlling their wafer sawing processes. As the modified process capability drops below the desired quality level, practitioners should take the proper actions immediately since the process does not meet their capability requirement.

As the subgroup size $n$ increases, the change in process variance has a higher probability of detection. For example, when the subgroup size $n$ increases to 25, the value of $A S_{50}$ is equal to 1.489 . Then, modified $\mathcal{C}_{p k}$ can be obtained as follows:

$$
\begin{aligned}
& \hat{C}_{p k} \\
& =\min \left\{\frac{U S L-\text { median }}{A S_{50}\left(\hat{F}_{99.865}-\text { median }\right)}, \frac{\text { median }-L S L}{A S_{50}\left(\text { median }-\hat{F}_{0.135}\right)}\right\} \\
& =\min \left\{\frac{80-45.365}{1.489(61.257-45.365)}, \frac{45.365-20}{1.489(45.365-36.148)}\right\} \\
& =1.464 .
\end{aligned}
$$


Increasing $n$ from 20 to 25 would increase the value of modified $\mathcal{C}_{p k}$ index from 1.395 to 1.464 , the corresponding manufacturing yield can be approximately assessed as $99.9971 \%$ and $99.9989 \%$, respectively, and the total number of defectives would be reduced.

\section{A. Discussion}

In this paper, we provide the accurate wafer sawing manufacturing yield assessment and present the accommodation $\left(\mathcal{A S}_{50}\right)$ of manufacturing yield for various subgroup $n$ and various $\alpha$ values. To mathematically demonstrate the relationship between $A S_{50}$ and scale parameter $(\beta)$, we obtain the probability of $\bar{X}_{n}$ when $X_{1}, \ldots, X_{n}$ is a random sample from Gamma $(\alpha, \beta)$. The $U C L$ and $L C L$ for $\bar{X}$ chart can be expressed as follows:

$$
U C L=\frac{\beta_{0}}{2 n} \chi_{1-\frac{\alpha}{2},\left(2 n \alpha_{0}\right)}^{2} \text { and } L C L=\frac{\beta_{0}}{2 n} \chi_{\frac{\alpha}{2},\left(2 n \alpha_{0}\right)}^{2} .
$$

Thus, we obtain

$$
1-\alpha=G_{\bar{X}}\left(\frac{\beta_{0}}{2 n} \chi_{1-\frac{\alpha}{2},\left(2 n \alpha_{0}\right)}^{2}\right)-G_{\bar{X}}\left(\frac{\beta_{0}}{2 n} \chi_{\frac{\alpha}{2},\left(2 n \alpha_{0}\right)}^{2}\right) .
$$

Let $X_{1}, \ldots, X_{n} \sim$ Gamma $\left(\alpha_{0}, \beta_{0}\right)$. We then obtain $X_{1}+$ $\cdots+X_{n} \sim \operatorname{Gamma}\left(n \alpha_{0}, \beta_{0}\right)$, and $\bar{X}=\left(X_{1}+\cdots+X_{n} / n\right) \sim$ Gamma $\left(n \alpha_{0}, \beta_{0} / n\right)$.

From the technique of transformation of random variables, we can obtain

$$
\frac{2 n}{\beta_{0}} \bar{X} \sim \operatorname{Gamma}\left(n \alpha_{0}, 2\right)=\chi_{\left(2 n \alpha_{0}\right)}^{2} .
$$

Consequently, we have the following results:

$$
\begin{aligned}
1-\alpha & =P\left(\chi_{\frac{\alpha}{2},\left(2 n \alpha_{0}\right)}^{2} \leq \frac{2 n}{\beta_{0}} \bar{X} \leq \chi_{1-\frac{\alpha}{2},\left(2 n \alpha_{0}\right)}^{2}\right) \\
& =P\left(\frac{\beta_{0}}{2 n} \chi_{\frac{\alpha}{2},\left(2 n \alpha_{0}\right)}^{2} \leq \bar{X} \leq \frac{\beta_{0}}{2 n} \chi_{1-\frac{\alpha}{2},\left(2 n \alpha_{0}\right)}^{2}\right) \\
& =G_{\bar{X}}\left(\frac{\beta_{0}}{2 n} \chi_{0.99865,\left(2 n \alpha_{0}\right)}^{2}\right)-G_{\bar{X}}\left(\frac{\beta_{0}}{2 n} \chi_{0.00135,\left(2 n \alpha_{0}\right)}^{2}\right) .
\end{aligned}
$$

The discussion presented above indicated that the evaluation of the accommodations $\left(\mathcal{A S}_{50}\right)$ would not be influenced by the scale parameter $(\beta)$. When we develop the accommodations $\left(\mathcal{A S} \mathcal{S}_{50}\right)$, without loss of generality, we can set $\beta=1$ to obtain the value of $A S_{50}$. The value of $A S_{50}$ would not be influenced by scale parameter $(\beta)$ of Gamma distribution. The result simplifies the complexity of estimating the distribution parameters. Consequently, the accuracy of manufacturing yield of sawing process can be improved.

Tool wear is a frequent and natural part in wafer sawing processes and is a systematic assignable cause. The fraction of defectives would rise as the tool deteriorates. Accordingly, ordinary capability measures become inaccurate because process data are contaminated by the assignable cause variation. In wafer sawing processes, one of the most important aspects of tool management is the tool replacement policy for a diamond blade. To determine the optimal tool replacement time of a diamond blade, we refer the interested reader to [17]. The authors presented an analytical approach using modified estimator of yield-based index $\left(\mathcal{C}_{v k}\right)$ under the condition of systematic assignable cause. To improve the accuracy of the capability assessment, they applied the linear regression technique to remove the variation due to an assignable cause from the measure of process capability. They considered that $u_{t}$ represents the mean and $\sigma_{r t}$ represents the variation (due to random causes only) of the process at time period $t$ in their modified capability assessment formula. They also replaced $u_{t}$ and $\sigma_{r t}$ by the estimators $\bar{X}_{t}$ and $\left[(n-2) M S E_{t} /(n-1)\right]^{1 / 2}$, respectively. $M S E_{t}$ is the mean square error associated with the regression equation $\hat{X}_{a i}=\hat{\alpha}_{a}+\hat{\beta}_{a} t_{a i}$, where $t_{a i}$ is the sequence number of the sampling unit and $\hat{\beta}_{a}$ denotes the linear change of tool wear given a unit change in time/production. Practitioners can use the modified capability evaluation formula to obtain manufacturing yield of wafer sawing processes more accurately, and make reliable decisions regarding the optimal tool replacement time.

\section{CONClusion}

In this paper, we considered the manufacturing yield assessment under undetected variance change conditions for Gamma wafer sawing processes. Sawing wastage is the essential parameter for controlling the manufacturing yield. Conventionally, applying the yield measure index method requires that the process is under stable condition and the process outcome follows normal distribution. However, in the Gamma wafer sawing processes, conventional manufacturing yield evaluation may mislead the practitioners to lose the critical chance to feedback the corrective actions on their cared processes due to overestimated manufacturing yield. Therefore, in this paper, we presented a yield measure index method incorporating the factor of undetected variance change to assess the manufacturing yield under real-world conditions. For illustration purpose, we considered a wafer sawing case taken from the factory located on the Science-based Industrial Park at Hsinchu, Taiwan. For Gamma processes, we mathematically showed the accommodation of manufacturing yield would not be affected by the scale parameter of a Gamma distribution. We also applied the Monte-Carlo simulation method to determine the control limits of $S^{2}$ chart and obtained the accommodations for various subgroup sizes and designated detection power. For small value of shape parameter of Gamma distribution, the required accommodation in the yield measure index formula is much greater than those for normal processes. Using the accommodated yield measure formula, the practitioners can determine the manufacturing yield more accurately. The detection powers of the $S^{2}$ chart under various subgroup sizes are tabulated. The results obtained could help the practitioners to make more correct decisions on what improved actions need to be taken in controlling Gamma wafer sawing processes.

\section{REFERENCES}

[1] L. Yang and J. Bernstein, "Reliability study of high-density EBGA packages using the Cu metallized silicon," IEEE Trans. Comp. Packag. Technol., vol. 31, no. 3, pp. 702-711, Sep. 2008.

[2] L. L. Mercado and V. Sarihan, "Evaluation of die edge cracking in flipchip PBGA packages," IEEE Trans. Comp. Packag. Technol., vol. 26, no. 4, pp. 719-723, Dec. 2003.

[3] M.-Y. Tsai and C. S. Lin, "Testing and evaluation of silicon die strength," IEEE Trans. Electron. Packag. Manuf., vol. 30, no. 2, pp. 106-114, Apr. 2007. 
[4] H. H. Jiun, I. Ahmad, A. Jalar, and G. Omar, "Effect of laminated wafer toward dicing process and alternative double pass sawing method to reduce chipping," IEEE Trans. Electron. Packag. Manuf., vol. 29, no. 1, pp. 17-24, Jan. 2006.

[5] C. H. Stapper, "Improved yield models for fault-tolerant memory chips," IEEE Trans. Comput., vol. 42, no. 7, pp. 872-881, Jul. 1993.

[6] I. Koren, Z. Koren, and C. H. Stapper, "A unified negative-binomial distribution for yield analysis of defect-tolerant circuits," IEEE Trans. Comput., vol. 42, no. 6, pp. 724-734, Jun. 1993.

[7] C. H. Stapper, "LSI yield modeling and process monitoring," IBM J. Res. Develop., vol. 44, nos. 1-2, pp. 112-117, Jan. 2000.

[8] T. Pyzdek, "Process capability analysis using personal computers," Qual. Eng., vol. 4, no. 3, pp. 419-440, 1992.

[9] K. C. Choi, K. H. Nam, and D. H. Park, "Estimation of capability index based on bootstrap method," Microelectron. Rel., vol. 36, no. 9, pp. 1141-1153, Sep. 1996.

[10] J.-W. Wang, C.-N. Wang, and W.-Y. Chen, "Three-step approach for wafer sawing lane inspection," Opt. Eng., vol. 48, no. 11, pp. 117204 1-117204-15, 2009.

[11] D. C. Montgomery, Introduction to Statistical Quality Control, 5th ed. New York: Wiley, 2005, pp. 337-343.

[12] W. L. Pearn, Y. C. Chang, and C. W. Wu, "Quality-yield measure for production processes with very low fraction defective," Int. J. Prod. Res., vol. 42, no. 23, pp. 4909-4925, 2004.

[13] V. E. Kane, "Process capability indices," J. Qual. Technol., vol. 18, no. 1, pp. 41-52, 1986.

[14] D. R. Bothe, "Statistical reason for the $1.5 \sigma$ shift," Qual. Eng., vol. 14, no. 3, pp. 479-487, 2002.

[15] Y. C. Hsu, W. L. Pearn, and P. C. Wu, "Capability adjustment for gamma processes with mean shift consideration in implementing six sigma program," Eur. J. Oper. Res., vol. 191, no. 2, pp. 517-529, Dec. 2008.

[16] S. Ross, A First Course in Probability Theory, 7th ed. New York: Academic, 2005.

[17] W. L. Pearn, Y.-C. Hsu, and C.-W. Wu, "Tool replacement for production with a low fraction of defectives," Int. J. Prod. Res., vol. 44, no. 12, pp. 2313-2326, 2006.

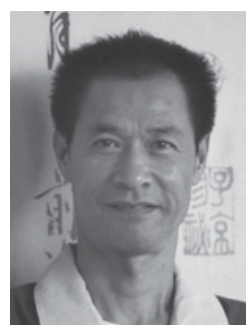

Wen-Lea Pearn received the Ph.D. degree in operations research from the University of Maryland, College Park.

$\mathrm{He}$ is a Professor of Operations Research and Quality Assurance at the National Chiao-Tung University (NCTU), Hsinchu, Taiwan, He was with Bell Laboratories, Murray Hill, NJ, as a Quality Research Scientist before joining the NCTU, and others. His current research interests include process capability, network optimization, and production management.
Dr. Pearn's publications have appeared in the Journal of the Royal Statistical Society, Series C, Journal of Quality Technology, European Journal of Operational Research, Journal of the Operational Research Society, Operations Research Letters, Omega, Networks, and the International Journal of Productions Research.

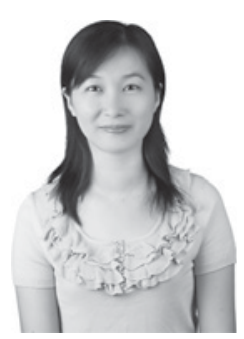

Yu-Ting Tai received the $\mathrm{Ph} . \mathrm{D}$. degree in industrial engineering and management from the National Chiao-Tung University, Hsinchu, Taiwan.

She is an Assistant Professor of the Department of Information Management, Kainan University, Taoyuan, Taiwan. Her current research interests include process capability indices, scheduling, and semiconductor manufacturing management.

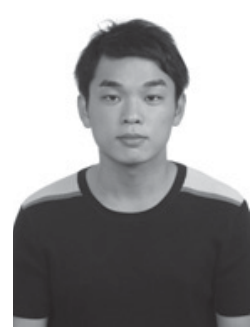

Kai-Bin Huang received the Ph.D. degree in industrial engineering and management from the National Chiao-Tung University, Hsinchu, Taiwan.

$\mathrm{He}$ is currently a Senior IT Engineer with the Engineering Information System Development Department, Ardentec Co. Ltd., Hsinchu. His current research interests include queueing theory, optimization theory, quality engineering, and applied statistics.

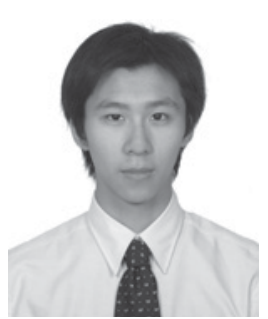

Pin-Lun Ku received the Masters degree in industrial engineering and management from the National Chiao-Tung University, Hsinchu, Taiwan.

$\mathrm{He}$ is currently a Material Control Engineer in the Project Service Division, CTCI Corporation, Bangkok, Thailand. His current research interests include process capability indices, quality engineering, and applied statistics. 